\title{
Infestation by Norwegian scabies
}

\author{
Chih-Chieh Chan MD, Sung-Jan Lin MD PhD, Ya-Chi Chan MS RN, Yi-Hua Liao MD PhD
}

Previously published at www.cmaj.ca on July 20, 2009.

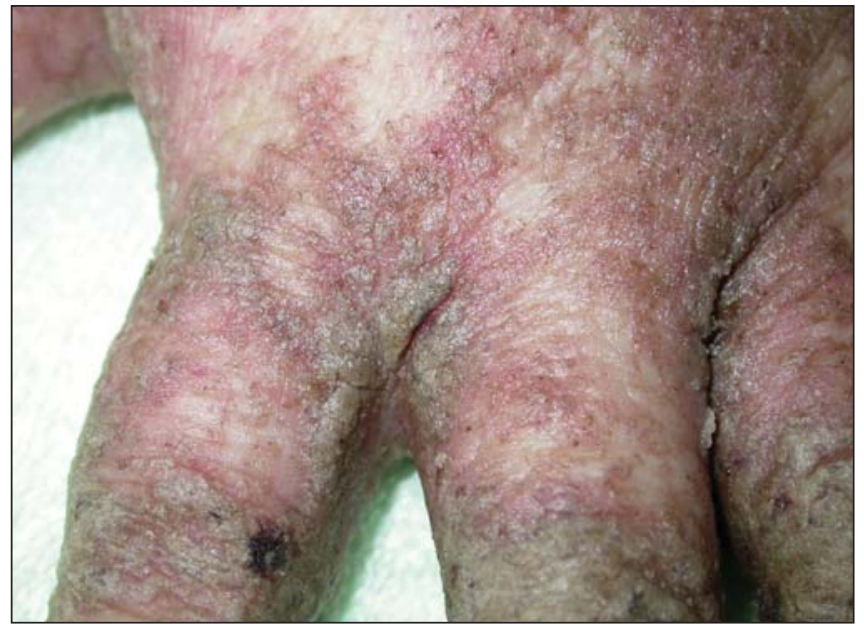

Figure 1: The hand of a 51-year-old woman with systemic lupus erythematosus, showing cutaneous greasy eruptions caused by Norwegian scabies.

A 51-year-old woman with an 8-year history of systemic lupus erythematosus was hospitalized with fever, refractory thrombocytopenia and generalized rash. The patient had previously undergone splenectomy. She had been taking azathioprine and prednisolone daily for more than 1 year. The rash had begun as itchy minor eruptions 3 months previously. Initially, it had been diagnosed as a psoriasiform dermatitis and was treated with topical corticosteroids. After 2 weeks of the corticosteroid therapy, the rash had worsened. The lesions became scaly, lichenified, crusted plaques on her hands (Figure 1), umbilicus, neck, upper back, armpits, inguinal folds and legs.

The distribution and appearance of these lesions was consistent with Norwegian (or crusted) scabies. Skin scrapings from the wrist and periumbilical plaques of the patient were examined microscopically. Numerous scabetic mites (Figure 2A), eggs and fecal pellets (also called scybala) (Figure 2B, arrows) were observed, which confirmed the diagnosis. The skin eruptions were treated successfully with application of topical $25 \%$ benzyl benzoate lotion for 1 week and removal of the crusts after softening of the scales with vegetable oil.

From the Department of Dermatology (Chih-Chieh Chan, Lin, Liao), National Taiwan University Hospital and Yun-Lin Branch, Taipei; the Institute of Biomedical Engineering (Chih-Chieh Chan, Lin), National Taiwan University, Taipei; and the Department of Nursing (Ya-Chi Chan), Taichung Armed Forces General Hospital, Taichung, Taiwan

Cite as CMAJ 209. DOI:10.1503/cmaj.081576
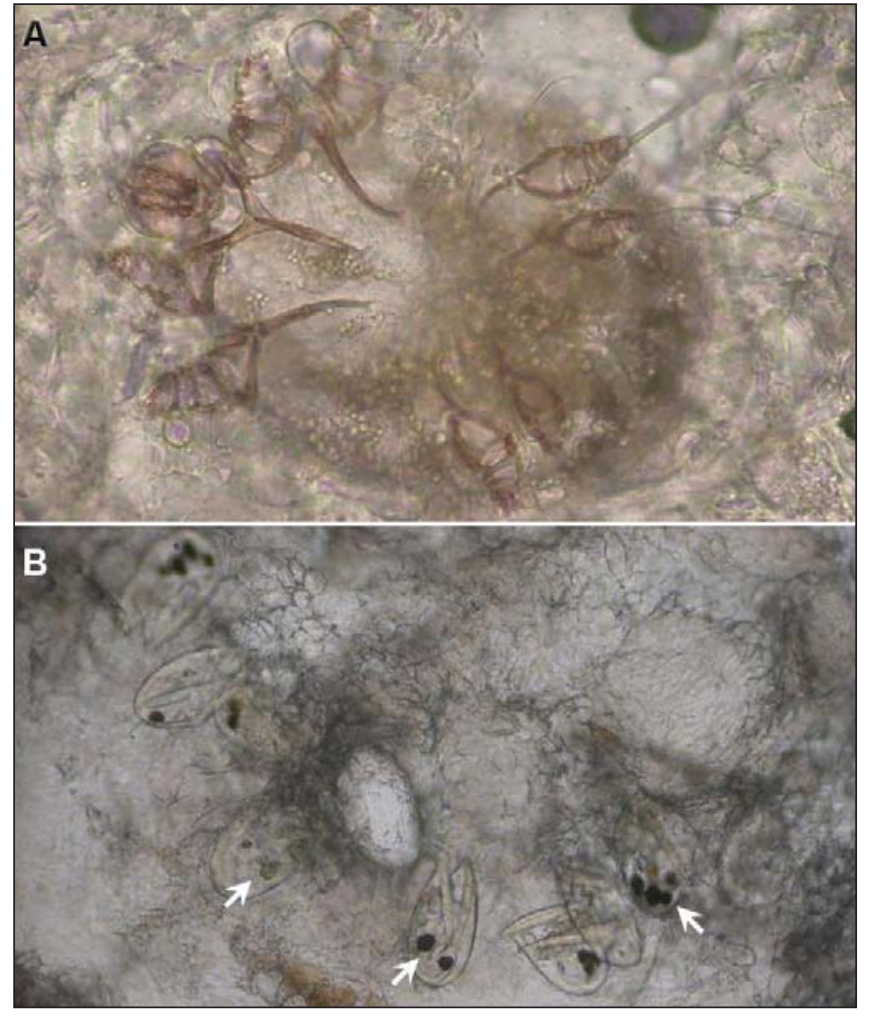

Figure 2: Microscopic image of skin scrapings showing scabetic mites (A), eggs and fecal pellets (B, arrows).

Crusted Norwegian scabies is highly contagious ${ }^{1}$ and may be misdiagnosed as chronic dermatitis. This uncommon, hyperkeratotic form of scabies infestation tends to occur in people who are immunosuppressed or elderly. Outbreaks can occur in institutions. ${ }^{2.3}$ Despite its high mite-load, Norwegian scabies may cause only mild discomfort, leading to delay in diagnosis and treatment. Topical scabicides are widely available for treating both classic and atypical scabies. An administration of 2 doses of oral ivermectin $(200 \mu \mathrm{g} / \mathrm{kg})$ given 2 weeks apart is very effective and may be used as a first-line therapy or in combination with topical agents. ${ }^{2}$

\section{REFERENCES}

1. Hsueh PR, Lin BH, Hwang CC, et al. Nosocomial outbreak of scabies. J Formos Med Assoc 1992;91:228-32.

2. Chosidow O. Clinical practices. Scabies. N Engl J Med 2006;354:1718-27.

3. Roberts LJ, Huffam SE, Walton SF, et al. Crusted scabies: clinical and immunological findings in seventy-eight patients and a review of the literature. $J$ Infect 2005;50:375-81. 